\title{
Comparing Postoperative Complications and Inflammatory Markers Using Total Intravenous Anesthesia Versus Volatile Gas Anesthesia for Pancreatic Cancer Surgery
}

\author{
Jose M Soliz, ${ }^{1,}$ Ifeyinwa C Ifeanyi, ${ }^{1}$ Mathew H Katz, ${ }^{2}$ Jonathan Wilks, ${ }^{1}$ Juan P Cata, ${ }^{1}$ Thomas McHugh, ${ }^{1}$ \\ Jason B Fleming, ${ }^{2}$ Lei Feng, ${ }^{3}$ Thomas Rahlfs, ${ }^{1}$ Morgan Bruno, ${ }^{2}$ and Vijaya Gottumukkala ${ }^{1}$ \\ ${ }_{1}^{1}$ Department of Anesthesiology and Perioperative Medicine, Houston, TX, USA \\ ${ }^{2}$ Department of Surgical Oncology, Houston, TX, USA \\ ${ }^{3}$ Department of Biostatistics, Houston, TX, USA \\ "Corresponding author: Jose M Soliz, MD, Department of Anesthesiology and Perioperative Medicine University of Texas M.D., Anderson Cancer Center, 1400 Holcombe Blvd, \\ Houston, TX 77030, E-mail: jsoliz@mdanderson.org
}

Received 2017 March 22; Revised 2017 May 23; Accepted 2017 August 07.

\begin{abstract}
Objectives: The objective of this study is to evaluate postoperative complications and inflammatory profiles when using a total intravenous anesthesia (TIVA) or volatile gas-opioid (VO) based anesthesia in patients undergoing pancreatic cancer surgery.

Methods: Design, retrospective propensity score matched cohort; Setting, major academic cancer hospital; Patients, all patients who had pancreatic surgery between November 2011 and August 2014 were retrospectively reviewed. Propensity score matched patient pairs were formed. A total of 134 patients were included for analysis with 67 matched pairs; Interventions, Patients were categorized according to type of anesthetic used (TIVA or VO). Patients in the TIVA group received preoperative celecoxib, tramadol, and pregabalin in addition to intraoperative TIVA with propofol, lidocaine, ketamine, and dexmedetomidine. The VO-group received a volatile-opioid based anesthetic; Measurements, demographic, perioperative clinical data, platelet lymphocyte ratios, and neutrophil lymphocyte ratios were collected. Complications were graded and collected prospectively and later reviewed retrospectively.

Results: Patients receiving TIVA were more likely to have no complication or a lower grade complication than the VO-group $(\mathrm{P}=$ 0.014). There were no differences in LOS or postoperative inflammatory profiles noted between the TIVA and VO groups.

Conclusions: In this retrospective matched analysis of patients undergoing pancreatic cancer surgery, TIVA was associated with lower grade postoperative complications. Length of hospital stay (LOS) and postoperative inflammatory profiles were not significantly different.
\end{abstract}

Keywords: Anesthesia, Cancer, Complications, Pancreas Surgery, TIVA, Volatile Anesthesia

\section{Background}

Despite advances in medicine and technology, pancreatic cancer surgery is still associated with high postoperativemorbidity. Severe postoperative complications have been reported in the literature to be $15 \%$ - 21\%after pancreatectomy $(1,2)$. The high rate of complications, while most usually not life threatening, could result in delays in return to intended oncologic therapy, in addition to increased length of stay (LOS)with an associated increase in medical costs (3-5). With this in mind, there has been an increasing interest in the role of anesthetic techniques in reducing postoperative complications and in the modulation of perioperative inflammation (6-8).

A volatile gas-opioid based anesthesia and total intravenous anesthesia (TIVA) are two of the common types of general anesthesia used for patients undergoing ma- jor abdominal surgery. The choice of anesthetic regimen is typically at the discretion of the attending anesthesiologist and may dependent on their training, experience with various anesthetic agents, or resources available at their practice site. In patients undergoing surgical resection as part of their cancer treatment, mitigating the perioperative inflammatory response and limiting the exposure to opioids thru the use of anesthetic techniques is of increasing interest (9). The role of perioperative inflammation,as measured by platelet-lymphocyte ratios (PLR) and neutrophil-lymphocyte ratios (NLR),has been correlated with both postoperative complications and worse oncologic outcomes (10-14). In a double-blinded randomized study, Sridhar et al found that intravenous lidocaine reduced the stress response and reduced opioid use after abdominal surgery (15). Likewise, the antiinflammatory properties of propofol (16), dexmedetomi- 
dine (17), and ketamine (18) have been studied. The role of preemptive analgesia through the use of non-narcotic analgesics and regional anesthesiahas yielded similar positive results on postoperative pain scores and inflammation (19-21). The anti-inflammatory and opioid reducing properties of these medications have led anesthesiologists to use them in concert through TIVA techniques, with the goal of diminishing the inflammatory response associated with surgery and possibly reduce postoperative complications.

The primary aim of this retrospective study is to evaluate the impact of TIVA on postoperative complications when compared to a volatile-opioid based regimen in patients undergoing pancreatic surgery. The secondary endpoints include analyzing postoperative inflammatory markers and hospital length of stay.

\section{Methods}

\subsection{Study Patient Selection}

After approval of the institutional review board (IRB\#: PA13-0223), all adult patients undergoing pancreatic cancer surgery between November 2011 and August 2014 were retrospectively reviewed. Patients were divided in twostudygroups based on the anesthetic regimen (TIVA vs. volatile-opioid anesthesia). Demographics, intraoperative data, postoperative complications and inflammatory markers (NLR and PLR) were collected and analyzed. Complications were prospectively identified and graded during the study period and retrospectively obtained from the institutional pancreatic surgical database. All adverse events that occurred within 90 days of surgery or any complication occurring after 90 days from surgery was included in analysis if the complication was directly related to the surgery (22). Adverse events were graded according to the modified accordion severity grading system $(23,24)$.

\subsection{Anesthetic Management}

The anesthetic technique selection was at the discretion of the attending anesthesiologist. Unless a contraindication existed, all patients were offered an epidural for postoperative analgesia. Epidural catheters were placed in the T7/8 or T8/9 interspaces under strict sterile conditions. In both study groups, epidural catheters were typically bolused at the beginning of surgery with hydromorphone 10 $\mathrm{mcg} / \mathrm{kg}$, and then infused with bupivacaine $0.075 \%$ with hydromorphone $5 \mathrm{mcg} / \mathrm{cc}$ at $8-10 \mathrm{cc} / \mathrm{hr}$ during surgery.

Patients assigned to the TIVA group received preoperative oral tramadol 300mg, pregabalin $75 \mathrm{mg}$, celexocib $400 \mathrm{mg}$, unless a contraindication existed. Anesthesia was maintained by total intravenous anesthesia with intraoperative infusions propofol, lidocaine, dexmedetomidine, and ketamine. Maintenance of anesthesia was titrated with the assistance of a bispectral index monitor (BISwith goal range 40 - 60). Goal directed fluid therapy (GDFT) was employed using The Vigileo ${ }^{\circledR}$ (Edwards Lifesciences, Irvine, CA, USA; EV1000) or LiDCOrapid ${ }^{\circledR}$ (LiDCO Group Plc, London, UK) with a target of maintaining stroke volume variation of less than 12.

Patients assigned to the volatile opioid (VO) group received a volatile gas based anesthetic and intravenous opioids. Typically, patients in the VO-group received 1 - $2 \mathrm{mg}$ IV midazolam preoperatively. Induction of anesthesia was typically performed using propofol $2-3 \mathrm{mg} / \mathrm{kg}$ and fentanyl $1-2 \mathrm{mcg} / \mathrm{kg}$. A volatile gas, typically desflurane (3\% - 7\%), was used for anesthesia maintenance. Intravenous narcotics using fentanyl (50 - $100 \mathrm{mcg}$ boluses) and/or hydromorphone (0.5 - $2 \mathrm{mg}$ boluses) were used for analgesia. GDFT was used at the not routinely used in this group.

\subsection{Propensity Matching and Statistical Analysis}

To adjust for selection bias in the observational study, a propensity score matched analysis was created from the eligible patients. The patient pairs were matched based on patient age, gender, epidural use, Charlson comorbidity index (25), type of surgery, and length of surgery. A Chisquare test was used to evaluate the association between two categorical variables. Wilcoxon rank sum test was used to evaluate the difference in a continuous variable between patient groups. The repeated measures models were fitted to evaluate the anesthesia technique effect and time effect on inflammatory markers, PLR, and NLR. A P value lower than 0.05 was considered statistically significant. Statistical software SAS 9.3 (SAS, Cary, NC) and S-Plus 8.2 (TIBCO Software Inc., Palo Alto, CA) were used for all the analyses.

\section{Results}

\subsection{Patient and Clinical Characteristics}

A total of 366 eligible patient cases were initially identified. Sixty-eight patients were assigned to the TIVA group and 298 assigned to the VO-group. After propensity score matching, 67 matched pairs, a total of 134 total patients, were identified for further analysis. Demographics and clinical characteristics of the matched patient pairs are summarized in Table 1 . All patients in both study groups received an epidural for intraoperative use and postoperative analgesia. There were no major epidural related complications (hematoma or abscess) in either study group. Preoperative hemoglobin was similar (TIVA: $13 \pm 1.64 \mathrm{~g} / \mathrm{dL}$ vs. $12.79 \pm 1.93 \mathrm{~g} / \mathrm{dL} ; \mathrm{P}=0.394)$. Patients in the VO group had slightly larger blood loss $(783 \pm 820 \mathrm{~mL})$ compared to the TIVA group ( $533 \pm 335 \mathrm{~mL} ; \mathrm{P}=0.186)$, though this was not 
statistically significant. The VO-group was more likely to be transfused intraoperativelywith packedred blood cells $(\mathrm{P}=$ $0.004)$ and fresh frozen plasma $(\mathrm{P}=0.042)$ than the subjects in the TIVA group. There were no differences in postoperative transfusions $(\mathrm{P}=0.594)$.The urinary output was significantly lower in the VO group $(571 \pm 331 \mathrm{~mL})$ than in the TIVA group $(775 \pm 523 \mathrm{~mL} ; \mathrm{P}=0.03)$.

\subsection{Perioperative Inflammatory Markers (NLR and PLR)}

There were no statistical differences $(P>0.05)$ in the NLR or PLR between the study groups at any time point both preoperatively and postoperatively (Figures 1 and 2 respectively).

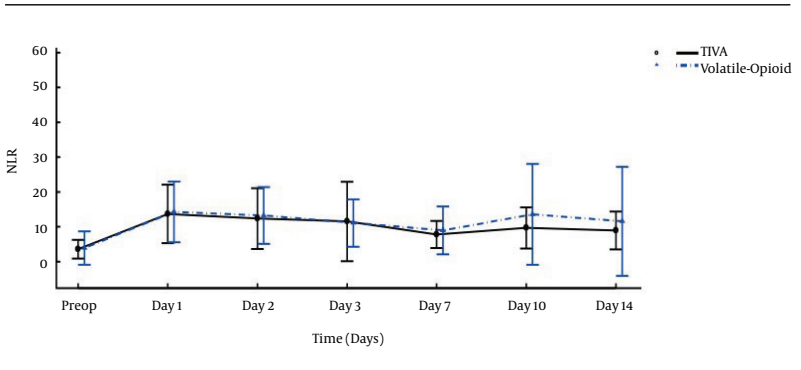

Figure 1. Average NLR by Anesthetic Technique

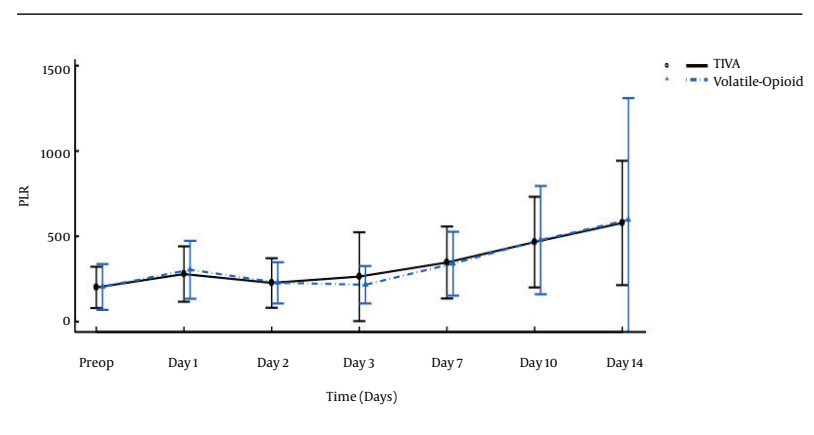

Figure 2. Average PLR by Anesthetic Technique

\subsection{Length of Hospital Stay and Postoperative Complications}

The mean LOS for the TIVA-group (11.3 \pm 4.5 days) was 1 day shorter than in the VO-group (12.9 \pm 5.9 days), although this difference was not statistically significant $(\mathrm{P}=$ 0.1; Table 1). Patients in the TIVA group had fewer postoperative adverse events within 90 days of surgery than in the VO group, but not statistically significant. Briefly, 51 (78.5\%) of the TIVA group patients compared to 57 (87.7\%) in the VO-group $(\mathrm{P}=0.161)$ had an adverse event within 90 days of surgery. There were no statistical differences in complications by organ system identified (Table 2). The most common complication by organ system in both groups was gastrointestinal complications (TIVA: 39 (60\%) vs. VO: 46 (70.8\%), $\mathrm{P}=0.197)$. Gastrointestinal complication included pancreatic leak, delayed gastric emptying, gastrojejunostomy leak, hepaticojejunostomy leak, duodenojejunostomy leak, ileus, chyle leak, and unclassified GI complications.

When graded by the modified Accordion Grading Severity System there were no differences $(P=0.084)$ in complications by severity grade (Table 2 ). However, patients in the TIVA-group were more likely to have no complication or a low-grade (grades 1 or 2 ) complication (Table 2) compared to the VO-group $(\mathrm{P}=0.014)$. The calculated average severity score for complicated patients was also similar between the study groups $(P=0.303)$. The difference in calculated postoperative morbidity index (PMI) (TIVA: 0.227 vs. VO: $0.28, P=0.095)$ did not reach statistical difference. Hospital readmission rates were comparable between the study groups (TIVA: 16 (24.6\%) vs. VO: 12 (18.5\%); P $=0.393$ ).

\section{Discussion}

The choice of the optimal anesthetic regimen should strike a balance between providing adequate pain relief, minimizing complications and length of hospital stay, as well as potentially suppressing inflammatory pathways that could mediate cancer recurrence. Multimodal analgesia consisting of regional anesthesia and an opiate sparing technique has been proven effective in improving short term post-operative outcomes (27). In our study, patients who received TIVA were more likely to have no postoperative complication or low-grade complications compared to those patients who received volatile-based anesthesia. These findings however did not correlate with a decreased length of stay. In addition, there was no difference in postoperative inflammatory profiles as measured by NLR or PLR between the study groups.

The concept that the NLR and PLR are biomarkers of immune suppression, systemic inflammation, and a proangiogenic state is well studied in the literature (28-30). These values, NLR and PLR, obtained from common laboratory studies served as markers of inflammation in our analysis. In a study by Haruki et al, the preoperative NLR was independently associated with worse long term outcomes after pancreaticoduodenectomy for carcinoma of the ampulla of vater (31). Similarly, preoperative NLR was associated with disease free survival after curative resection for pancreatic adenocarcinoma (32). As such, efforts to mitigate inflammation in the perioperative setting are imperative.

Ramirez et al. demonstrated that at clinically relevant concentrations of intravenous lidocaine, natural killer cell 
Table 1. Patient Demographics and Clinical Characteristics ${ }^{\mathrm{a}}$

\begin{tabular}{|c|c|c|}
\hline Variables & $\operatorname{TIVA} \operatorname{group}(N=67)$ & VO-Group $(N=67)$ \\
\hline Age & $61.3 \pm 12.6$ & $61.7 \pm 12.7$ \\
\hline \multicolumn{3}{|l|}{ Gender } \\
\hline Female & $23(34.3 \%)$ & $23(34.3 \%)$ \\
\hline Male & $44(65.7)$ & $44(65.7)$ \\
\hline \multicolumn{3}{|l|}{ ASA classification, No. (\%) } \\
\hline 2 & $6(9)$ & $8(11.9)$ \\
\hline 3 & $61(91)$ & $58(86.6)$ \\
\hline 4 & $0(0)$ & $1(1.5 \%)$ \\
\hline BMI & $27.1 \pm 4.3$ & $27.9 \pm 5.2$ \\
\hline Charlson comorbidity index & $3.3 \pm 1.4$ & $3.3 \pm 1.6$ \\
\hline Preoperative Hemoglobin & $13 \pm 1.64$ & $12.79 \pm 1.93$ \\
\hline \multicolumn{3}{|l|}{ Type of surgery, No. (\%) } \\
\hline Pancreaticoduodenectomy & $49(73.1)$ & $48(71.6)$ \\
\hline Distal pancreatectomy & $14(20.9)$ & $16(23.9)$ \\
\hline Total pancreatectomy & $2(3)$ & $1(1.5)$ \\
\hline Combined whipple $^{\mathrm{b}}$ & $2(3)$ & $2(3)$ \\
\hline Surgery length, min & $449 \pm 141$ & $444 \pm 169$ \\
\hline EBL, $\mathbf{m L}$ & $533 \pm 335$ & $783 \pm 820$ \\
\hline Median (range) & $450(100-2500)$ & $550(100-4950)$ \\
\hline Crystalloid, mL & $2765 \pm 824$ & $2674 \pm 1262$ \\
\hline Colloid, mL & $1219 \pm 583$ & $1378 \pm 764$ \\
\hline Urine output, $\mathbf{m L}^{\mathrm{c}}$ & $775 \pm 523$ & $571 \pm 331$ \\
\hline \multicolumn{3}{|l|}{ Intraoperative Transfusions, No. (\%) } \\
\hline Packed red blood cells ${ }^{c}$ & $8(11.9)$ & $22(32.8)$ \\
\hline Fresh frozen plasma ${ }^{\mathrm{C}}$ & $0(0)$ & $4(6)$ \\
\hline Platelets & $1(1.5)$ & $1(1.5)$ \\
\hline Length of stay, days & $11.3 \pm 4.5$ & $12.9 \pm 5.9$ \\
\hline Median (range) & $10(4-25)$ & $11(5-36)$ \\
\hline
\end{tabular}

Abbreviations: ASA, American Society of Anesthesiologists Physical Classification; BMI, Body Mass Index; EBL, Estimated Blood Loss.

${ }^{a}$ Values are expressed as means and standard deviation, or number and percentages unless specified otherwise.

${ }^{\mathrm{b}}$ Combined whipple: patients received whipple operation in addition to either nephrectomy or thoracotomy.

${ }^{\mathrm{c}}$ Denotes statistically significant $\mathrm{p}$ values $(\mathrm{P}<0.05)$

function was enhanced (33). Intraoperative propofol and dexmedetomidine have been shown to decrease intraoperative levels of interleukins 6 and $8(34,35)$. Likewise, the intraoperative use of ketamine has been shown reduce levels of interleukin 6 for several days after a bolus induction dose (36). In our study, a TIVA technique was planned to include multiple medications that have proven antiinflammatory properties. We proposed that by combining these medications in concert, the additive effect would result in a more favorable postoperative inflammatory pro- file. However, our results do not support this proposition. Perhaps the use of epidural analgesia in all patients may have contributed to lack of differences in NLR and PLR profiles between the study groups. In a prospective randomized trial of patients undergoing Ivor-Lewis esophagectomy, patients who received epidural analgesia had a lower postoperative inflammatory response (37). Similar results were reported in knee arthroplasty and spine surgery (38, 39). The use of epidural analgesia may potentially result in greater postoperative anti-inflammatory effects than from 
Table 2. Postoperative Complications According to Treatment Group

\begin{tabular}{|c|c|c|c|}
\hline Variables & TIVA-Group $^{\mathbf{a}}$ & VO-Group $^{a}$ & P Value \\
\hline Patients with adverse events, No. (\%) & $51(78.5)$ & $57(87.7)$ & 0.161 \\
\hline \multicolumn{4}{|c|}{ Complication by organ system (number of patients), No. (\%) } \\
\hline Gastrointestinal & $39(60)$ & $46(70.8)$ & 0.197 \\
\hline Cardiovascular & $1(1.5)$ & $1(1.5)$ & 1.000 \\
\hline Pulmonary & $4(6.2)$ & $5(7.7)$ & 1.00 \\
\hline Renal & 0 & $3(4.6)$ & 0.244 \\
\hline Hematologic & $4(6.2)$ & $3(4.6)$ & 1.000 \\
\hline Infectious & $27(41.5)$ & $29(44.6)$ & 0.723 \\
\hline Neurologic & 0 & $4(6.2)$ & 0.119 \\
\hline Metabolic & $2(3.1)$ & $1(1.5)$ & 1.000 \\
\hline Bleeding & $12(18.5)$ & $13(20)$ & 0.833 \\
\hline Other & $3(4.6)$ & $8(12.4)$ & 0.206 \\
\hline Accordion severity grade ${ }^{c}$, No. (\%) & & & 0.084 \\
\hline No complication & $14(21.5)$ & $8(12.3)$ & \\
\hline 1 & $13(20)$ & $8(12.3)$ & \\
\hline 2 & $24(36.9)$ & $22(33.8)$ & \\
\hline 3 & $9(13.8)$ & $20(30.8)$ & \\
\hline 4 & $2(3.1)$ & $6(9.2)$ & \\
\hline 5 & $2(3.1)$ & $1(1.5)$ & \\
\hline 6 & $1(1.5)$ & $0(0)$ & \\
\hline \multicolumn{4}{|l|}{ Accordion severity grade, No. (\%) } \\
\hline No Complication, Grades 1,or 2 & $51(78.5)$ & $38(58.5)$ & 0.014 \\
\hline Grades $3,4,5$, or 6 & $14(21.5)$ & $27(41.5)$ & \\
\hline Average severity score $^{d}$ & $0.289 \pm 0.184$ & $0.323 \pm 0.145$ & 0.303 \\
\hline Postoperative morbidity index (PMI) ${ }^{\mathrm{e}}$ & $0.227 \pm 0.202$ & $0.28 \pm 0.172$ & 0.095 \\
\hline Hospital readmission, No. (\%) & $16(24.6)$ & $12(18.5)$ & 0.393 \\
\hline \multicolumn{4}{|c|}{$\begin{array}{l}\text { Abbreviation: PMI, Postoperative Morbidity Index. } \\
\text { a Two patients each in the TIVA and VO-groups were excluded due to incomplete postoperative complication data (all were combination whipple cases). } \\
\text { borgan system complications include not highest-grade complications. } \\
{ }^{c} \text { Modified Accordion severity grading system (23), highest grade only. } \\
{ }^{d} \text { Average severity score calculated by summing the weights of the highest grade complication for each patient divided by the number of patients who had a complication } \\
\text { in each study group. }\end{array}$} \\
\hline
\end{tabular}

the use of the aforementioned TIVA medications.

The authors do note limitations to this study. The study was performed retrospectively, so selection biases can exist between the study groups. The authors attempted to minimize selection bias with a propensity score model matching similar patient cases. However, there could have been unaccounted factors that may have influenced the outcomes that were not measured or studied. In addition, the choice of anesthetic regimen (TIVA or VO) was at the discretion of the attending anesthesiologist. A noted dif- ference in the study groups is intraoperative blood transfusion with PRBC and FFP being greater in the VO-group despite statistical similarity in EBL. This may be the result of different transfusion triggers amongst anesthesiologists who perform the VO and TIVA techniques. While adverse events were obtained retrospectively for purposes of this study, the collection of adverse events occurred prospectively and was recorded in the pancreatic surgical database. Despite showing a difference in no complication or lower-grade complication in the TIVA group, perhaps 
the sample size was inadequate to detect differences in PMI or severity scores between the groups.

In conclusion, patients who received TIVA were more likely to have no complication or lower grade complication when compared to a volatile gas-opioid based anesthetic regimen for pancreatic cancer surgery. However, when graded for severity, the average severity score and postoperative morbidity index was similar. In addition, use TIVA did not result in a difference in postoperative inflammatory profiles. This study demonstrates that future randomized control trials are warranted to further study the optimal anesthetic regimens associated with improved outcomes after pancreatic cancer surgery.

\section{Acknowledgments}

The authors would like to acknowledge Priya Sahai, M.D., Alejandra Nava, M.D., and Vinny Singh, M.D. for their contribution in data collection for this study.

\section{Footnote}

Funding/Disclosures: This work was supported in part by the Cancer Center Support Grant (NCI Grant P30 CA016672). The authors report no conflicts of interest with regards to this study.

\section{References}

1. Okabayashi T, Shima Y, Iwata J, Morita S, Sumiyoshi T, Kozuki A, et al. Is a surgical approach justified for octogenarians with pancreatic carcinoma? Projecting surgical decision making for octogenarian patients. Am J Surg. 2016;212(5):896-902. doi: 10.1016/j.amjsurg.2015.12.033. [PubMed: 27262755].

2. Yamashita S, Ishizawa T, Ichida A, Kaneko J, Aoki T, Sakamoto Y, et al. Advantages and Disadvantages of Prophylactic Abdominal Drainage in Distal Pancreatectomy. World J Surg. 2016;40(5):1226-35. doi: 10.1007/s00268-015-3399-4. [PubMed: 26768889].

3. Aloia TA, Zimmitti G, Conrad C, Gottumukalla V, Kopetz S, Vauthey JN. Return to intended oncologic treatment (RIOT): a novel metric for evaluating the quality of oncosurgical therapy for malignancy. J Surg Oncol. 2014;110(2):107-14. doi: 10.1002/jso.23626. [PubMed: 24846705].

4. Kim BJ, Caudle AS, Gottumukkala V, Aloia TA. The Impact of Postoperative Complications on a Timely Return to Intended Oncologic Therapy (RIOT): the Role of Enhanced Recovery in the Cancer Journey. Int Anesthesiol Clin. 2016;54(4):e33-46. doi: 10.1097/AIA.0000000000000113. [PubMed: 27623128].

5. Crippa S, Salvia R, Falconi M, Butturini G, Landoni L, Bassi C. Anastomotic leakage in pancreatic surgery. $H P B$ (Oxford). 2007;9(1):8-15. doi: 10.1080/13651820600641357. [PubMed: 18333107].

6. Owusu-Agyemang P, Cata JP, Fournier KF, Zavala AM, Soliz J, Hernandez $\mathrm{M}$, et al. Evaluating the Impact of Total Intravenous Anesthesia on the Clinical Outcomes and Perioperative NLR and PLR Profiles of Patients Undergoing Cytoreductive Surgery with Hyperthermic Intraperitoneal Chemotherapy. Ann Surg Oncol. 2016;23(8):2419-29. doi: 10.1245/s10434-016-5176-5. [PubMed: 26975738].
7. Markovic-Bozic J, Karpe B, Potocnik I, Jerin A, Vranic A, Novak-Jankovic $\mathrm{V}$. Effect of propofol and sevoflurane on the inflammatory response of patients undergoing craniotomy. BMC Anesthesiol. 2016;16:18. doi 10.1186/s12871-016-0182-5. [PubMed: 27001425].

8. Sun B, Wang J, Bo L, Zang Y, Gu H, Li J, et al. Effects of volatile vs. propofol-based intravenous anesthetics on the alveolar inflammatory responses to one-lung ventilation: a meta-analysis of randomized controlled trials. J Anesth. 2015;29(4):570-9. doi:10.1007/s00540015-1987-y. [PubMed: 25716536].

9. Snyder GL, Greenberg S. Effect of anaesthetic technique and other perioperative factors on cancer recurrence. $\mathrm{Br} J$ Anaesth. 2010;105(2):106-15. doi: 10.1093/bja/aeq164. [PubMed: 20627881].

10. Spolverato G, Maqsood H, Kim Y, Margonis G, Luo T, Ejaz A, et al. Neutrophil-lymphocyte and platelet-lymphocyte ratio in patients after resection for hepato-pancreatico-biliary malignancies. I Surg Oncol. 2015;111(7):868-74. doi: 10.1002/jso.23900. [PubMed: 25865111].

11. Shirai Y, Shiba H, Sakamoto T, Horiuchi T, Haruki K, Fujiwara Y, et al. Preoperative platelet to lymphocyte ratio predicts outcome of patients with pancreatic ductal adenocarcinoma after pancreatic resection. Surgery. 2015;158(2):360-5. doi: 10.1016/j.surg.2015.03.043. [PubMed: 26032829].

12. Kordzadeh A, Malietzis G, Browne T, Prionidis I, Panayiotopoulos YP Neutrophil to lymphocyte ratio (NLR) of five predicts 30-day morbidity in ruptured abdominal aortic aneurysms (rAAA): a retrospective cohort study. Int J Surg. 2015;15:45-8. doi: 10.1016/j.ijsu.2015.01.013. [PubMed: 25641718].

13. Que Y, Qiu H, Li Y, Chen Y, Xiao W, Zhou Z, et al. Preoperative plateletlymphocyte ratio is superior to neutrophil-lymphocyte ratio as a prognostic factor for soft-tissue sarcoma. BMC Cancer. 2015;15:648. doi: 10.1186/s12885-015-1654-6. [PubMed: 26432433].

14. Pang Q, Zhang LQ, Wang RT, Bi JB, Zhang JY, Qu K, et al. Platelet to lymphocyte ratio as a novel prognostic tool for gallbladder carcinoma. World JGastroenterol. 2015;21(21):6675-83. doi:10.3748/wjg.v21.i21.6675. [PubMed: 26074706].

15. Sridhar P, Sistla SC, Ali SM, Karthikeyan VS, Badhe AS, Ananthanarayanan $\mathrm{PH}$. Effect of intravenous lignocaine on perioperative stress response and post-surgical ileus in elective open abdominal surgeries: a double-blind randomized controlled trial. ANZ J Surg. 2015;85(6):425-9. doi: 10.1111/ans.12783. [PubMed: 25078385].

16. Ma L, Wu X, Chen W, Fujino Y. Propofol has anti-inflammatory effects on alveolar type II epithelial cells. Acta Anaesthesiol Scand. 2010;54(3):362-9. doi: 10.1111/j.1399-6576.2009.02127.x. [PubMed: 19764911].

17. Bulow NM, Colpo E, Pereira RP, Correa EF, Waczuk EP, Duarte MF, et al. Dexmedetomidine decreases the inflammatory response to myocardial surgery under mini-cardiopulmonary bypass. Braz J Med Biol Res. 2016;49(4):e4646. doi: 10.1590/1414-431X20154646. [PubMed: 26909786].

18. Yang C, Jiang RY, Shen J, Hong T, Liu N, Ding LC, et al. Ketamine attenuates the lipopolysaccharide-induced inflammatory response in cultured N2a cells. Mol Med Rep. 2013;8(1):217-20. doi: 10.3892/mmr.2013.1465. [PubMed: 23660699].

19. Chen JQ, Wu Z, Wen LY, Miao JZ, Hu YM, Xue R. Preoperative and postoperative analgesic techniques in the treatment of patients undergoing transabdominal hysterectomy: a preliminary randomized trial BMC Anesthesiol. 2015;15:70. doi: 10.1186/s12871-015-0046-4. [PubMed: 25943183].

20. Zawar BP, Mehta Y, Juneja R, Arora D, Raizada A, Trehan N. Nonanalgesic benefits of combined thoracic epidural analgesia with general anesthesia in high risk elderly off pump coronary artery bypass patients. Ann Card Anaesth. 2015;18(3):385-91. doi: 10.4103/09719784.159810. [PubMed: 26139745].

21. Sidiropoulou I, Tsaousi GG, Pourzitaki C, Logotheti H, Tsantilas D, Vasilakos DG. Impact of anesthetic technique on the stress response elicited by laparoscopic cholecystectomy: a randomized trial. $J$ Anesth. 2016;30(3):522-5. doi: 10.1007/s00540-016-2148-7. [PubMed 26882921]. 
22. Schwarz L, Bruno M, Parker NH, Prakash L, Mise Y, Lee JE, et al. Active Surveillance for Adverse Events Within 90 Days: The Standard for Reporting Surgical Outcomes After Pancreatectomy. Ann Surg Oncol. 2015;22(11):3522-9. doi: 10.1245/s10434-015-4437-z. [PubMed: 25694246].

23. Strasberg SM, Linehan DC, Hawkins WG. The accordion severity grading system of surgical complications. Ann Surg. 2009;250(2):177-86. doi: 10.1097/SLA.0b013e3181afde41. [PubMed: 19638919].

24. Vollmer CJ, Lewis RS, Hall BL, Allendorf JD, Beane JD, Behrman SW, et al. Establishing a quantitative benchmark for morbidity in pancreatoduodenectomy using ACS-NSQIP, the Accordion Severity Grading System, and the Postoperative Morbidity Index. Ann Surg. 2015;261(3):527-36. doi: 10.1097/SLA.0000000000000843. [PubMed 25268299].

25. Quan H, Li B, Couris CM, Fushimi K, Graham P, Hider P, et al. Updating and validating the Charlson comorbidity index and score for risk adjustment in hospital discharge abstracts using data from 6 countries. Am J Epidemiol. 2011;173(6):676-82. doi: 10.1093/aje/kwq433. [PubMed: 21330339].

26. Strasberg SM, Hall BL. Postoperative morbidity index: a quantitative measure of severity of postoperative complications. J Am Coll Surg. 2011;213(5):616-26. doi: 10.1016/j.jamcollsurg.2011.07.019. [PubMed: 21871822].

27. Cata JP, Lasala J, Bugada D. Best practice in the administration of analgesia in postoncological surgery. Pain Manag. 2015;5(4):273-84. doi: 10.2217/pmt.15.21. [PubMed: 26072922].

28. Zhang H, Zhang L, Zhu K, Shi B, Yin Y, Zhu J, et al. Prognostic Significance of Combination of Preoperative Platelet Count and NeutrophilLymphocyte Ratio (COP-NLR) in Patients with Non-Small Cell Lung Cancer: Based on a Large Cohort Study. PLoS One. 2015;10(5):e0126496. doi: 10.1371/journal.pone.0126496. [PubMed: 25950176].

29. Neal CP, Cairns V, Jones MJ, Masood MM, Nana GR, Mann CD, et al. Prognostic performance of inflammation-based prognostic indices in patients with resectable colorectal liver metastases. Med Oncol. 2015;32(5):144. doi: 10.1007/s12032-015-0590-2. [PubMed: 25807934].

30. Raffetti E, Donato F, Castelli F, Maggiolo F, Carosi G, Quiros-Roldan E. The predictive role of NLR and PLR for solid non-AIDS defining cancer incidence in HIV-infected subjects: a MASTER cohort study. Infect Agent Cancer. 2015;10:34. doi: 10.1186/s13027-015-0032-y. [PubMed: 26442127].
31. Haruki K, Shiba H, Horiuchi T, Shirai Y, Iwase R, Fujiwara Y, et al. Neutrophil to Lymphocyte Ratio Predicts Therapeutic Outcome After Pancreaticoduodenectomy for Carcinoma of the Ampulla of Vater. Anticancer Res. 2016;36(1):403-8. [PubMed: 26722073].

32. Garcea G, Ladwa N, Neal CP, Metcalfe MS, Dennison AR, Berry DP. Preoperative neutrophil-to-lymphocyte ratio (NLR) is associated with reduced disease-free survival following curative resection of pancreatic adenocarcinoma. World J Surg. 2011;35(4):868-72. doi:10.1007/s00268011-0984-z. [PubMed: 21312035].

33. Ramirez MF, Tran P, Cata JP. The effect of clinically therapeutic plasma concentrations of lidocaine on natural killer cell cytotoxicity. Reg Anesth Pain Med. 2015;40(1):43-8. doi: 10.1097/AAP.0000000000000191. [PubMed: 25469757].

34. Corcoran TB, Engel A, Sakamoto H, O'Shea A, O'Callaghan-Enright S, Shorten GD. The effects of propofol on neutrophil function, lipid peroxidation and inflammatory response during elective coronary artery bypass grafting in patients with impaired ventricular function. BrJAnaesth. 2006;97(6):825-31. doi:10.1093/bja/ael270. [PubMed: 17032661].

35. Li B, Li Y, Tian S, Wang H, Wu H, Zhang A, et al. Anti-inflammatory Effects of Perioperative Dexmedetomidine Administered as an Adjunct to General Anesthesia: A Meta-analysis. Sci Rep. 2015;5:12342. doi: 10.1038/srep12342. [PubMed: 26196332].

36. Dale O, Somogyi AA, Li Y, Sullivan T, Shavit Y. Does intraoperative ketamine attenuate inflammatory reactivity following surgery? A systematic review and meta-analysis. Anesth Analg. 2012;115(4):934-43. doi: 10.1213/ANE.ob013e3182662e30. [PubMed: 22826531].

37. Fares KM, Mohamed SA, Hamza HM, Sayed DM, Hetta DF. Effect of thoracic epidural analgesia on pro-inflammatory cytokines in patients subjected to protective lung ventilation during Ivor Lewis esophagectomy. Pain Physician. 2014;17(4):305-15. [PubMed: 25054390].

38. Chloropoulou P, Iatrou C, Vogiatzaki T, Kotsianidis I, Trypsianis G, Tsigalou C, et al. Epidural anesthesia followed by epidural analgesia produces less inflammatory response than spinal anesthesia followed by intravenous morphine analgesia in patients with total knee arthroplasty. Med Sci Monit. 2013;19:73-80. [PubMed: 23353589].

39. Ezhevskaia AA, Prusakova Zh B, Maksimova LP, Sholkina MN, Balmusova EA, Ovechkin AM. [Effects of epidural anesthesia on stressinduced immune supression during major corrective spine surgery]. Anesteziol Reanimatol. 2014;59(6):4-9. [PubMed: 25831694]. 